\title{
Mathematical modelling on particle diffusion in fluidised beds and dense turbulent two-phase flows
}

\author{
R. Groll \\ Center of Applied Space Technology and Microgravity, \\ Germany
}

\begin{abstract}
Volume-fraction weighted and Reynolds averaged momentum transport equations are solved in an Euler/Euler approach to numerically simulate the turbulent, dispersed two-phase flow in a two-dimensional channel and a three-dimensional conic diffuser flow. Particular attention is given to the modelling of turbulent diffusion and particle wall interaction, assuming local equilibrium, but introducing individual terms for particle/fluid drag interaction, particle collisions and trajectory crossings. These influences have been quantified in terms of partial viscosities, a restitution power and a turbulence structure parameter. Boussinesq approximations have been used for each phase and the formulation of their interaction was provided in the framework of the eddy-viscosity modelling concept.

Keywords: two-phase flow, particle diffusion, particle collision, fluidised bed.
\end{abstract}

\section{Introduction}

The momentum transport equation includes a turbulent diffusion term, which characterises motions that are not resolved by the convective term. This turbulent diffusion depends on the turbulent kinetic energy and the turbulence characterising Eulerian time scale. Based on "Csanadys Approximation" [3], the time scale of the dispersed phase is coupled with the turbulent time scale of the continuous phase. This time scale quantifies the diffusion intensity [5] and is influenced by the drag interaction of the particles with the viscous gas phase and inter-particle collisions $[4,9]$. The fluctuation of a filtered variable $\phi$ is written:

$$
\{\phi\}^{k}=\phi-<\phi>^{k} \text {. }
$$


The index $k$ stands for $C$ (continuous phase) or $D$ (dispersed phase). The following negation notation is used here, i.e. $\bar{C}=D, \bar{D}=C$. The drag force relaxation time scale $\tau_{p}^{k}$ depends on the particle Reynolds number, based on the velocity difference between the continuous and the dispersed phase [12], see e.g. Crowe et al. (1998) [2]. The equation terms are described with the help of an averaging operator $<.>^{k}[11]$ :

$$
<u_{i}^{k}>^{l}=\frac{\overline{\alpha^{l} u_{i}^{k}}}{\bar{\alpha}^{l}} ; \quad u_{i}^{k}=<u_{i}^{k}>^{l}+\left\{u_{i}^{k}\right\}^{l} .
$$

Summarising the different diffusion generating components to a combined particle-turbulence shear stress model the diffusion of dispersed particles is predicted very well, especially inside turbulent shear layers.

\section{Transport equations}

Modelling the diffusion character of particle flows transported in a viscous carrier phase the diffusion is not dominated by the eddy dissipation as it is in turbulent shear flows in a continuous gas phase. This dispersed phase diffusion is characterised by unsteady drag influences and crossing trajectory characteristics defined by the correlation of velocities of the continuous phase and the dispersed phase itself. The Reynolds averaged, volume-fraction weighted momentum transport equations for the continuous and the dispersed phase read:

$$
\begin{aligned}
& \frac{\partial}{\partial t}\left(\rho^{k} \bar{\alpha}^{k}<u_{i}^{k}>^{k}\right)+\frac{\partial}{\partial x_{j}}\left(\rho^{k} \bar{\alpha}^{k}<u_{i}^{k}>^{k}<u_{j}^{k}>^{k}\right) \\
& =-\bar{\alpha} \frac{\partial p^{C}}{\partial x_{i}}+\bar{\alpha} \frac{\partial \sigma_{i j}^{k}}{\partial x_{j}}+\rho^{k} \frac{\bar{\alpha}^{k}}{\tau_{p}^{k}}\left(<u_{i}^{\bar{k}}>^{\bar{k}}-<u_{i}^{k}>^{k}\right) \\
& \quad+\rho^{k} \bar{\alpha}^{k} g_{i}-\frac{\partial}{\partial x_{j}}\left(\rho^{k} \bar{\alpha}^{k}<\left\{u_{i}^{k}\right\}^{k}\left\{u_{j}^{k}\right\}^{k}>^{k}\right)
\end{aligned}
$$

Collision and crossing trajectory terms were approximated by Grad [4] and Csanady [3]. The pressure gradient of the continuous phase is equivalent to the lift force. And the sum would be zero in a hydrostatic case. $\sigma_{i j}^{k}$ defines the viscous stresses inside the phase $k$. Because of the solid character of the dispersed phase $\sigma_{i j}^{D}$ is zero.

The underlying turbulence model for both phases is based on the Boussinesq analogy, employing eddy viscosity as the model quantity, whose formulation was provided in the framework of the standard $k-\epsilon$ modelling concept. $k^{C}$ defines the turbulent kinetic energy of the continuous phase. The corresponding variable of the dispersed phase $k^{D}$ describes the particle velocity variance at a given position. 


$$
\begin{aligned}
& \frac{\partial}{\partial t}\left(\rho^{k} \bar{\alpha}^{k} k^{k}\right)+\frac{\partial}{\partial x_{j}}\left(\rho^{k} \bar{\alpha}^{k} k^{k}<u_{j}^{k}>^{k}\right) \\
& \quad=\frac{\partial}{\partial x_{j}}\left(\bar{\alpha}^{k}\left(\mu^{k}+\rho^{k} \frac{v_{t}^{k}}{\sigma_{k}^{k}}\right) \frac{\partial k^{k}}{\partial x_{j}}\right)-\rho^{k} \bar{\alpha}^{k}<\left\{u_{i}^{k}\right\}^{k}\left\{u_{j}^{k}\right\}^{k}>^{k} \frac{\partial<u_{i}^{k}>^{k}}{\partial x_{j}} \\
& \text { with } \sigma_{k}^{C}=1,0 \text { and } \sigma_{k}^{D}=2.5
\end{aligned}
$$

The transport equations for the turbulent kinetic energy $k^{k}$ and its dissipation rate $\epsilon_{t}^{k}$ differ from those for a single-phase flow by several additional production terms and the modified dissipation. This total turbulent kinetic energy loss $\epsilon_{\alpha}^{k}$ in the present model arises from relative drag and particle collision processes.

$$
\begin{aligned}
\frac{\partial}{\partial t}\left(\rho^{k} \bar{\alpha}^{k} \epsilon_{t}^{k}\right)+\frac{\partial}{\partial x_{j}}\left(\rho^{k} \bar{\alpha}^{k} \epsilon_{t}^{k}<u_{j}^{k}>^{k}\right) \\
=\frac{\partial}{\partial x_{j}}\left(\bar{\alpha}^{k}\left(\mu^{k}+\rho^{k} \frac{v_{t}^{k}}{\sigma_{\epsilon}^{k}}\right) \frac{\partial \epsilon_{t}^{k}}{\partial x_{j}}\right)-C_{1} \frac{\epsilon_{t}^{k}}{k^{k}} \rho^{k} \bar{\alpha}^{k}<\left\{u_{i}^{k}\right\}^{k}\left\{u_{j}^{k}\right\}^{k}>^{k} \frac{\partial<u_{i}^{k}>^{k}}{\partial x_{j}} \\
\quad-C_{2} \rho^{k} \bar{\alpha}^{k} \frac{\epsilon_{t}^{k} \epsilon_{\alpha}^{k}}{k^{k}}+C_{3} \frac{\epsilon_{t}^{k}}{k^{k}} \rho^{k} \frac{\bar{\alpha}^{k}}{\tau_{p}^{k}}\left(q^{k}-2 k^{k}\right) \\
\quad \text { with } \sigma_{\epsilon}^{C}=1.3, C_{1}=1.44, C_{2}=1.92, C_{3}=1.2
\end{aligned}
$$

Because of the different phase velocities inside the drag relation term a general formulation is needed for the complimentary index of $k$. The velocity covariance $q^{k}=<\left\{u_{i}^{k}\right\}^{k}\left\{u_{i}^{\bar{k}}\right\}^{\bar{k}}>^{k}$ of He and Simonin (1993) [5] represents the trace of the velocity vector correlation tensor of both phases:

$$
\begin{aligned}
& \frac{\partial}{\partial t}\left(\rho^{k} \bar{\alpha}^{k} q^{k}\right)+\frac{\partial}{\partial x_{j}}\left(\rho^{k} \bar{\alpha}^{k} q^{k}<u_{j}^{k}>^{k}\right) \\
& =\frac{\partial}{\partial x_{j}}\left(\rho^{k} \bar{\alpha}^{k} v_{\alpha}^{k} \frac{\partial q^{k}}{\partial x_{j}}\right)+\rho^{k} \frac{\bar{\alpha}^{k}}{\tau_{p}^{k}}\left(2 Z^{k} k^{k}+2 k^{\bar{k}}-\left(1+Z^{k}\right) q^{k}\right) \\
& \quad-\rho^{k} \bar{\alpha}^{k}<\left\{u_{i}^{k}\right\}^{k}\left\{u_{j}\right\}^{k}>^{k} \frac{\partial<u^{k}>^{k}}{\partial x_{j}}-\rho^{k} \bar{\alpha}^{k}<\left\{u_{i}^{\bar{k}}\right\}^{\bar{k}}\left\{u_{j}^{\bar{k}}\right\}^{\bar{k}}>^{k} \frac{\partial<u^{\bar{k}}>^{\bar{k}}}{\partial x_{j}} \\
& \quad \text { with } Z^{k}=\frac{\rho^{k} \bar{\alpha}^{k}}{\rho^{\bar{k}} \bar{\alpha}^{\bar{k}}},
\end{aligned}
$$

which completes the present three-equation model for each of both phases. Velocity correlations, representing the turbulent momentum diffusion, are modelled by the following Boussinesq approximations:

$$
\begin{aligned}
- & <\left\{u_{i}^{k}\right\}^{k}\left\{u_{j}^{k}\right\}^{k}>^{k} \\
& =-\frac{2}{3} k^{k} \delta_{i j}+v_{t}^{k}\left(\frac{\partial<u_{i}^{k}>^{k}}{\partial x_{j}}+\frac{\partial<u_{j}^{k}>^{k}}{\partial x_{i}}-\frac{2}{3} \frac{\partial<u_{l}^{k}>^{k}}{\partial x_{l}} \delta_{i j}\right)
\end{aligned}
$$




$$
\begin{aligned}
- & <\left\{u_{i}^{k}\right\}^{k}\left\{u_{j}^{\bar{k}}\right\}^{\bar{k}}>^{k} \\
& =-\frac{1}{3} q^{k} \delta_{i j}+v_{\alpha}^{k}\left(\frac{\partial<u_{i}^{k}>^{k}}{\partial x_{j}}+\frac{\partial<u_{j}^{k}>^{k}}{\partial x_{i}}-\frac{2}{3} \frac{\partial<u_{l}^{k}>^{k}}{\partial x_{l}} \delta_{i j}\right) .
\end{aligned}
$$

Modelling the viscous continuous phase the eddy viscosity is modelled based on eddy dissipation relating components. Because of the phase interaction influence on the turbulent diffusion of both phases an additional transport equation for the velocity covariance has to be defined. The diffusion coefficients of momentum and turbulent transport equations are given by the characteristic diffusion time scale $\tau_{\alpha}^{k}$ :

$$
v_{t}^{k}=\tau_{\alpha}^{k} \cdot \frac{2}{3} k^{k} \quad \text { and } \quad v_{\alpha}^{k}=\tau_{\alpha}^{k} \cdot \frac{2}{3} q^{k}
$$

To close the present formulation of particle and carrier gas phase motion this time scale has to be modelled. Based on this new kind of particle diffusion modelling also equilibrium-turbulence boundary conditions are modified by the influences of inter-particle collisions and phase-interactions of dispersed and continuous phase.

\section{Diffusion modelling}

Based on this model of momentum diffusion, which depends on the velocity gradients of the diffusing phase, the characteristic diffusion time scales have to be defined by the velocity correlation and its associated loss rate $\epsilon_{\alpha}^{k}$. Local equilibrium describes the equivalence of production and loss of turbulent kinetic energy. Assuming $\partial / \partial x_{1} \approx 0$ yields an expression $(i=1, j=2)$ for the nondiagonal element of the Reynolds stress tensor.

$$
\begin{aligned}
\epsilon_{\alpha}^{k} & =-<\left\{u_{1}^{k}\right\}^{k}\left\{u_{2}^{k}\right\}^{k}>^{k} \frac{\partial<u_{1}^{k}>^{k}}{\partial x_{2}} \\
\Rightarrow-<\left\{u_{1}^{k}\right\}^{k}\left\{u_{2}^{k}\right\}^{k}>^{k} & =\frac{\left(<\left\{u_{1}^{k}\right\}^{k}\left\{u_{2}^{k}\right\}^{k}>^{k}\right)^{2}}{\epsilon_{\alpha}^{k}} \frac{\partial<u_{1}^{k}>^{k}}{\partial x_{2}}
\end{aligned}
$$

With the definition of the turbulence structure parameter $C_{\alpha}^{k}$ of the phase $k$

$$
\begin{gathered}
C_{\alpha}^{k}=-\frac{<\left\{u_{1}^{k}\right\}^{k}\left\{u_{2}^{k}\right\}^{k}>^{k}}{\frac{2}{3} k^{k}} \\
\Rightarrow \quad v_{t}^{k} \frac{\partial<u_{1}^{k}>^{k}}{\partial x_{2}}=-<\left\{u_{1}^{k}\right\}^{k}\left\{u_{2}^{k}\right\}^{k}>^{k}=\frac{\left(\frac{2}{3} C_{\alpha}^{k} k^{k}\right)^{2}}{\epsilon_{\alpha}^{k}} \frac{\partial<u_{1}^{k}>^{k}}{\partial x_{2}}
\end{gathered}
$$

the turbulent viscosity is calculated using the turbulent kinetic energy $k^{k}$, its loss rate and the turbulence structure parameter. Based on the diffusion definition (eq.9) 
Table 1: Restitution power and turbulence loss components.

\begin{tabular}{|c|l||c|c|c|c|}
\hline$\gamma$ & model & $\pi_{\gamma}^{C}$ & $\epsilon_{\gamma}^{C}$ & $\pi_{\gamma}^{D}$ & $\epsilon_{\gamma}^{D}$ \\
\hline \hline$\beta$ & Jones et al. & $\frac{2}{3} \beta \epsilon_{\beta}^{C}$ & $\epsilon_{t}^{C}$ & 0. & 0. \\
\hline$d$ & Csanady & 0. & 0. & $\frac{2}{3} \frac{k^{D}}{\tau_{\alpha}^{C}} \sqrt{1+C_{\beta}^{C}\left(\xi_{r}^{C}\right)^{2}}$ & 0. \\
\hline$p$ & Wallis & $\frac{2}{3} \frac{2 k^{C}-q^{C}}{\tau_{p}^{C}}$ & $\frac{2 k^{C}-q^{C}}{\tau_{p}^{C}}$ & $\frac{2}{3} \frac{2 k^{D}-q^{D}}{\tau_{p}^{D}}$ & $\frac{2 k^{D}-q^{D}}{\tau_{p}^{D}}$ \\
\hline$c$ & Jenkins et al. & 0. & 0. & $\frac{\sigma_{c}^{D}}{\tau_{p}^{D}} \frac{2}{3} k^{k}$ & $\frac{k^{k}}{\tau_{c}^{D}}\left(1-\left(e_{c}^{D}\right)^{2}\right)$ \\
\hline
\end{tabular}

the turbulent time scale is also defined by these values:

$$
v_{t}^{k}=-\frac{2}{3} \tau_{\alpha}^{k} k^{k} \Rightarrow \tau_{\alpha}^{k}=\frac{v_{t}^{k}}{\frac{2}{3} k^{k}}=\frac{2}{3}\left(C_{\alpha}^{k}\right)^{2} \frac{k^{k}}{\epsilon_{\alpha}^{k}}
$$

With the definition of the restitution power $\pi_{\alpha}^{k}$, the turbulent viscosity is determined by the turbulent kinetic energy and the restitution power.

$$
\pi_{\alpha}^{k}=\frac{2}{3} \frac{k^{k}}{\tau_{\alpha}^{k}}=\frac{\epsilon_{\alpha}^{k}}{\left(C_{\alpha}^{k}\right)^{2}} \Rightarrow-<\left\{u_{1}^{k}\right\}^{k}\left\{u_{2}^{k}\right\}^{k}>^{k}=\frac{\left(\frac{2}{3} k^{k}\right)^{2}}{\pi_{\alpha}^{k}} \frac{\partial<u_{1}^{k}>^{k}}{\partial x_{2}}
$$

The power $\pi_{\alpha}^{k}$ describes the restitution of turbulent shear forces based on the dissipation and structure of turbulence and reduces the turbulent diffusion.

This restitution power consists of the partial powers defined by four different effects (see Table 1), which are described in the following subsections with the indices $\beta, p, c$ and $d$. The total turbulence loss rate $\epsilon_{\alpha}^{k}$ is given by the sum of individual loss rates $\left(\epsilon_{\gamma}^{k}\right.$, see Table 1):

$$
\epsilon_{\alpha}^{k}=\sum_{\gamma} \epsilon_{\gamma}^{k}=\epsilon_{\beta}^{k}+\epsilon_{d}^{k}+\epsilon_{p}^{k}+\epsilon_{c}^{k}
$$

The different diffusion rates and turbulence loss rates are induced by the viscous turbulent shear stress $\left(\pi_{\beta}^{k}, \epsilon_{\beta}^{k}\right.$ : Jones et al. [7]), crossing trajectory effects $\left(\pi_{d}^{k}, \epsilon_{d}^{k}\right.$ : Csanady [3]), drag forces $\left(\pi_{p}^{k}, \epsilon_{p}^{k}\right.$ : Schiller and Naumann [12]) and collision terms $\left(\pi_{c}^{k}, \epsilon_{c}^{k}\right.$ : Jenkins and Richman [9]). Adding together these influences, the new restitution power term of the turbulent diffusion is modelled.

$$
\pi_{\alpha}^{k}=\sum_{\gamma} \pi_{\gamma}^{k}=\pi_{\beta}^{k}+\pi_{d}^{k}+\pi_{p}^{k}+\pi_{c}^{k}
$$



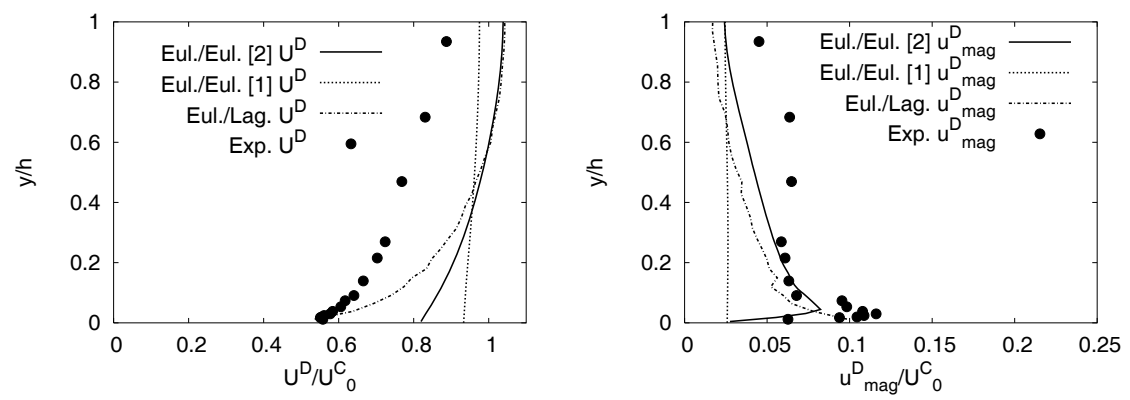

Figure 1: Normalised stream-wise particle mean velocity $U^{D}$ and normalised standard deviation $u_{m a g}^{D}$ of the particle velocity magnitude in the fullydeveloped, particle-laden channel flow.

Simulating the general restitution power $\pi_{\alpha}^{k}$ and energy loss $\epsilon_{\alpha}^{k}$ the resulting turbulence structure parameter $C_{\alpha}^{k}$ is computed in the following way:

$$
\pi_{\alpha}^{k}=\frac{2}{3} \frac{k^{k}}{\tau_{\alpha}^{k}}=\frac{\epsilon_{\alpha}^{k}}{\left(C_{\alpha}^{k}\right)^{2}} \Rightarrow C_{\alpha}^{k}=\sqrt{\frac{\epsilon_{\alpha}^{k}}{\pi_{\alpha}^{k}}}
$$

The turbulent time scale $\tau_{\alpha}^{k}$ depends on the sum of all diffusion rates:

$$
\tau_{\alpha}^{k}=\frac{\frac{2}{3} k^{k}}{\pi_{\beta}^{k}+\pi_{d}^{k}+\pi_{p}^{k}+\pi_{c}^{k}}
$$

This way of calculation yields a deterministic method to compute the turbulent time scale, which is needed for the calculation of the general turbulent viscosity of both phases (eq.12).

\section{Computational results and discussion}

This model was validated using experimental data of $70 \mu \mathrm{m}$ copper particles in a fully-developed channel flow ([10]; experimental results). The results using the present model (Eul./Eul. [2]) were also compared with the results obtained by an Euler/Lagrange scheme (Eul./Eul.) [6,8] and a well-known Euler/Eulerian diffusion approach (Eul./Eul. [1]) [5, 11].

Gravity acts in the positive $\mathrm{x}$-axis direction. The channel flow Reynolds number, based on channel height $(2 \mathrm{~h}=40 \mathrm{~mm})$ and single phase channel centreline velocity $\left(U_{0}^{C}=10.5 \mathrm{~m} / \mathrm{s}\right)$ is $R e_{2 h}=27600$. The flow is regarded as fully developed after 125 channel heights and at this position it is assumed that the particle velocity and particle turbulence has reached an asymptotic state. The copper particles have a density of $\rho^{D}=8800 \mathrm{~kg} / \mathrm{m}^{3}$ and a diameter of $D_{p}=$ $70 \mu \mathrm{m}$. The inlet mass loading of particles is $Z_{0}^{D}=10 \%$ and the parameter of 

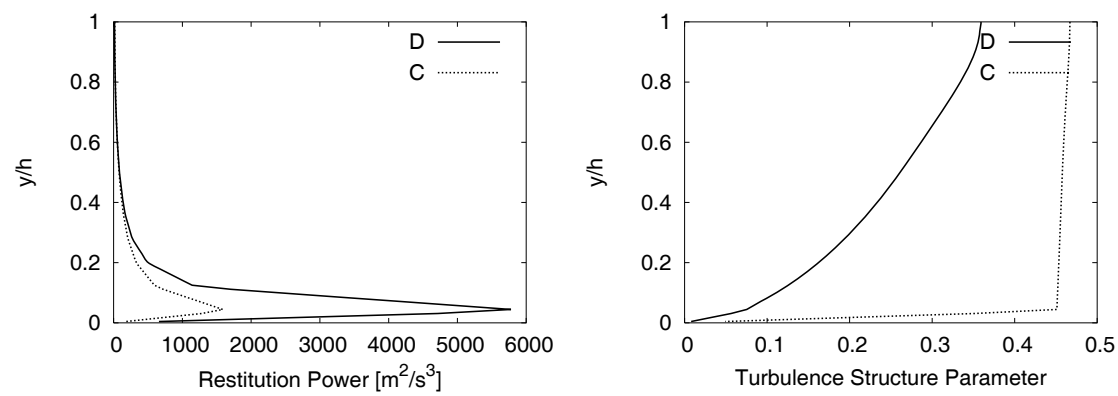

Figure 2: Turbulent kinetic energy loss rate $\epsilon_{\alpha}^{k}$ and turbulence restitution power $\pi_{\alpha}^{k}$ of the dispersed phase (D) and the continuous phase (C).

elasticity is $e_{c}^{D}=0.90$. The results obtained using the present method were also compared with the computational results obtained by an Euler/Lagrange scheme [8].

Comparing the present model (Eul./Eul. [2]) with a standard particle diffusion model (Eul./Eul. [1]), the velocity and the standard deviation of the velocity magnitude of the present model agree better with the Euler/Lagrangian results. Because of the assumed isotropy of the present model, the standard deviation of the stream-wise particle velocity does not agree as well with the predicted standard deviation of the particle velocity magnitude.

The characteristic model values describing diffusion: the restitution power $\pi_{\alpha}^{k}$ and the turbulence structure parameter $C_{\alpha}^{k}$ are shown in Fig. 2. The dissipation loss of the continuous phase and the restitution power of the dispersed phase dominate over the respective values of the other phase. As expected the turbulence structure parameter of the dispersed phase CD decreases near the wall, because of the decreasing ratio $\sqrt{\epsilon_{\alpha}^{D} / \pi_{\alpha}^{D}}$ against the nearly constant turbulence structure parameter of the continuous phase $C_{\alpha}^{C}$. Comparing the positions of these restitution maxima of the momentum diffusion with local minima of the turbulence structure parameter $C_{\alpha}^{D}$ (Fig. 2, right) near the wall the difference between the different kinds of diffusion, with and without viscous turbulence dissipation, in dispersed and continuous phase are shown.

Validating this new particle diffusion model a test case of Bohnet and Triesch [1] has been chosen. Simulating a fluidised bed with a particle loading $Z=1$ inside a rising vertical diffuser flow. Glass particles are used approaching the dispersed phase. The half-cone angle of the conic diffuser is $6^{\circ}$ and the outlet/inlet diameter ratio is $D_{2} / D_{1}=1.45$. The particle laden flow enters the diffuser part of the channel after a distance, which is long enough to develop a fully turbulent flow.

Analysing the velocity profiles (Fig. 3) local loading, gas and particle velocities are shown at entrance and exit of the diffuser. The gas phase inlet-boundary condition is a velocity block profile of $U_{0}=26.0 \mathrm{~m} / \mathrm{s}$. The symmetry line is at $x=0$. Inside the diffuser the gas velocity decreases and is overtaken by the particles. The particle velocity decreases during the relaxation process upside the 

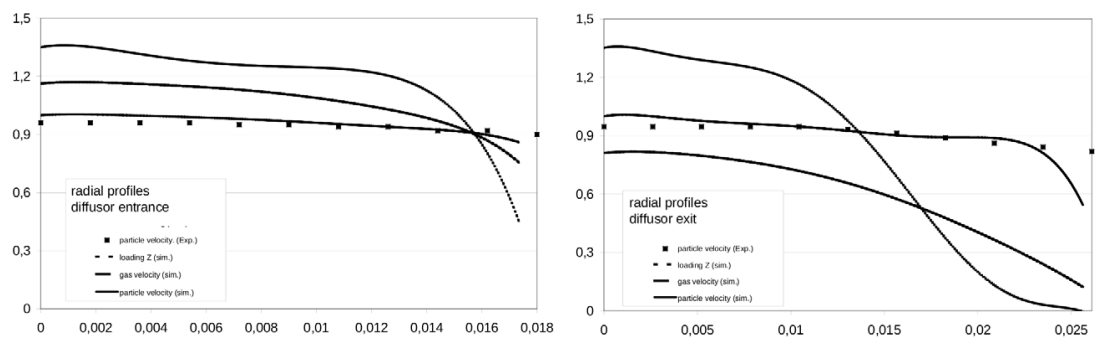

Figure 3: Normalised radial profiles of axial gas velocity, particle velocity and loading in comparison with experimental results.

diffuser. The particle loading has a similar maximum at the exit of the diffuser. Also this maximum decrease during the homogenising diffusion process upside the diffuser. The numerical data are compared with experimental data of Bohnet and Triesch [1].

\section{Conclusions}

Using this kind of diffusion blending, simulations are able to give better results for turbulent wall layers of the dispersed phase, including their turbulence production. The prediction of turbulent particle diffusion is limited by the quality of modelling of the momentum diffusion and the turbulence production of the dispersed phase.

Compared to classical equilibrium models, which solve an additional differential equation for the energy loss, coming up to the dissipation rate in viscous systems, the energy loss of the dispersed phase is given by algebraic equations. The turbulence structure parameter remains nearly constant in the dissipative systems examined here. So the ratio of restitution and dissipation power of the involved sub-models keeps also nearly constant.

For the not viscous, dispersed phase the turbulence structure parameter decreases corresponding to the high restitution and the locally low momentum diffusion inside the wall layer. This characteristic behaviour is based on the additional restitution power without the corresponding loss rate induced by crossing trajectories effects. Basically the added modelling of the turbulence structure parameter influences the production of particle velocity variance near the wall in that kind that the gradients of the stream-wise particle velocity agree with the measurement data.

\section{References}

[1] Bohnet A. and Triesch O., Influence of particles on fluid turbulence, Chem. Eng. Techn. 26:1254ff., 2003. 
[2] Crowe C.T., Sommerfeld M., and Tsuji Y., Multiphase flows with droplets and particles. CRC Press LLC, Florida, 1998.

[3] Csanady G.T., Turbulent diffusion of heavy particles in the atmosphere. J. Atm. Sc. 20:201ff., 1963.

[4] Grad H., On the kinetic theory on rarefied gases. Communications on Pure and Applied Mathematics 2(4):331ff., 1949.

[5] He J., and Simonin O., Non-equilibrium prediction of the particle-phase stress tensor in vertical pneumatic conveying. ASME-FED : Gas-Solid Flows 166: $253,1993$.

[6] Huber N., and Sommerfeld M., Characterization of the cross-particle concentration distribution in pneumatic conveying systems. Powder Techn. 79:191ff., 1994.

[7] Jones W. P., and B. E. Launder, The prediction of laminarization with a twoequation model of turbulence. Int. J. Heat and Mass Transf. 15:301ff., 1972.

[8] Kohnen G., ber den Einfluss der Phasenwelchselwirkungen bei turbulenten Zweiphasenstrmungen und deren numerische Erfassung in der Euler/Lagrange Betrachtungsweise (tranl. On the influence of phase interactions of turbulent two-phase flows and their numerical description in the Euler/Lagrangian approach), Shaker Verlag, Aachen, Germany, 1997.

[9] Jenkins J. T., M. W. Richman, Grads 13-moment-system for a dense gas of inelastic spheres. Arch. Ration. Mech. Anal. 87:355ff., 1985.

[10] Kulick J. D., Fessler J.R., Eaton J.K., Particle response and turbulence modification in fully developed channel flow. J. Fluid Mech. 277:109ff., 1994.

[11] Politis S. 1989. Prediction of two-phase solid-liquid turbulent flow in stirred vessels, PhD Thesis, Imperial College London, 1989.

[12] Schiller L., A. Naumann, Über die grundlegenden Berechnungen bei der Schwerkraftaufbereitung (transl.: On the basic calculations in gravity conditioning), VDI-Journal 77:318ff., 1933. 